\title{
DESIGN DE OBJETOS DE APRENDIZAGEM DIGITAL DO PONTO DE VISTA DA USABILIDADE
}

\author{
Gustavo Henrique Mochiuti ${ }^{1}$, Vânia Cristina Pires Nogueira Valente ${ }^{2}$
}

\begin{abstract}
Technological resources and the media are contributing to socioeconomic development, including the education sector. Application of Digital Learning Objects support learning, serving as a tool for distance learning arrangements and classroom. The quality of design in instructional materials is one factor that implies the motivation of students and support their learning, within this context, the creation of educational interactive multimedia applications should excel the content and good usability, while facilitating user interaction. The aim of this paper was to establish parameters for the creation and management of Digital Learning Objects.
\end{abstract}

Index Terms — Digital Learning Objects, interactivity, usability

\section{INTRODUÇÃO}

Os recursos tecnológicos e os meios de comunicação vêm contribuindo para o desenvolvimento socioeconômico, incluindo o setor de educação. A aplicação de objetos de aprendizagem digital é um suporte ao aprendizado, servindo como ferramenta de auxilio de modalidades de ensino à distância e presencial.

A chave de acesso para o ensino telemático passa, necessariamente, pelo domínio do processo de conversão dos conteúdos escritos da cultura clássica e contemporânea, em mensagens e programas audiovisuais. $\mathrm{O}$ cuidado de escolher uma linguagem audiovisual apropriada para adaptar um conteúdo, escrito ou exposto oralmente, evita o risco de se transformar uma exposição presencial desanimada em uma mensagem eletrônica, ainda mais aborrecida. Podemos citar, por exemplo, o uso sem critérios de recursos audiovisuais de forma a cansar poluir e prejudicar a transmissão do conteúdo previamente elaborado.

"A qualidade do design nos materiais instrucionais é um dos fatores que implicam na motivação dos alunos e na facilitação do seu aprendizado”. [23]

Dentro desse contexto, a criação de aplicações multimídias educacionais interativas deve primar pelo conteúdo e boa usabilidade. [13] define usabilidade como um atributo de qualidade, que avalia o grau de facilidade de interação de algum dispositivo ou qualquer interface que possa ser operada por um usuário. Além disso, a palavra também se refere aos métodos para melhorar a facilidade de uso durante o processo de planejamento de uma interface.

$\mathrm{O}$ autor elenca cinco componentes que definem a usabilidade, apresentados no Quadro 1:

QUADRO I

COMPONENTES QUE DEFINEM A INTERATIVIDADE [13]

\begin{tabular}{ll}
\hline $\begin{array}{l}\text { Componente } \\
\text { Facilidade de } \\
\text { aprendizado }\end{array}$ & Descrição \\
\hline Eficiência de uso & $\begin{array}{l}\text { Uma vez aprendido a interagir, o usuário } \\
\text { deve ser apto a atingir altos níveis de } \\
\text { produtividade. }\end{array}$ \\
\hline $\begin{array}{l}\text { Facilidade de } \\
\text { memorização }\end{array}$ & $\begin{array}{l}\text { usuário casual precisa sem utilizá-lo, o } \\
\text { retornar ao sistema e realizar suas tarefas, } \\
\text { sem a necessidade de reaprender como } \\
\text { interagir com ele. }\end{array}$ \\
\hline Baixa taxa de erros & $\begin{array}{l}\text { O usuário deve ser capaz de operar o } \\
\text { sistema sem maiores transtornos, com } \\
\text { baixa taxa de erros. Caso estes ocorram, } \\
\text { o usuário deve ser capaz de se recuperar. } \\
\text { Erros grosseiros não devem ocorrer. }\end{array}$ \\
\hline $\begin{array}{l}\text { A interação deve ser agradável, de forma } \\
\text { que o usuário se sinta subjetivamente } \\
\text { satisfeito ao utilizar o sistema. }\end{array}$ \\
\hline
\end{tabular}

\section{OBJETOS DE APRENDIZAGEM DigITAL}

Compreende-se por Objeto de Aprendizagem Digital (OAD) uma ferramenta digital que auxilia a aprendizagem, incorporando elementos tecnológicos à práticas e teorias pedagógicas.

É sabido que as transformações ocorridas nos meios de comunicação foram impactantes no âmbito educacional e pedagógico: desde a criação da oralidade, ensino através da escrita, o livro como principal suporte, aos recursos computacionais e as atuais Tecnologias de Informação e Comunicação (TIC).

Desde o surgimento dos primeiros computadores, os educadores projetam a possibilidade do uso das máquinas como auxiliares de processo ensino-aprendizagem.

$\mathrm{Na}$ década de 50, Burrhus Frederi Skinner, psicólogo americano, desenvolveu o primeiro sistema computadorizado com fins pedagógicos, utilizando instruções programadas seguindo modelo behaviorista, cedendo lugar posteriormente à psicologia cognitiva com seus sistemas de tutores inteligentes, onde a construção do conhecimento é feita através do processamento da informação. Posteriormente, o construtivismo de Jean William Fritz Piaget, epistemólogo suíço, foi implantado com facilidade como metodologia educacional, através de softwares simuladores que envolvem possibilidades de desenvolvimento de hipóteses, teses e análise de resultados, criando uma espécie de simuladores que permitem interação do usuário.

Com a inserção de computadores mais poderosos e ferramentas de comunicação que permitem a troca de informações mais rápida, novos modelos pedagógicos foram implantados utilizando formas sofisticadas de apoio ao ensino, como sistemas de autoria e de

\footnotetext{
${ }^{1}$ Curso de Design - Faculdade de Arquitetura, Artes e Comunicação - Universidade Estadual Paulista Julio de Mesquita Filho - UNESP - Bauru, SP, Brasil, ghakusho13@gmail.com

${ }_{2}^{2}$ Departamento de Artes e Representação Gráfica - Faculdade de Arquitetura, Artes e Comunicação - Universidade Estadual Paulista Julio de Mesquita Filho - UNESP - Bauru, SP, Brasil, vania@ faac.unesp.br
} 
hipertexto, buscando a convergência de mídia e de periféricos e utilizando também os novos dispositivos como tablets, ipad, celulares, móbile, etc.

Diante dessa necessidade, em 2001 o Ministério da Educação (MEC) criou o projeto Rede Interativa Virtual de Educação (Rived) com o objetivo de criar materiais digitais e disponibilizá-los em um repositório, para serem utilizados pelos professores nas escolas públicas.

Para formulação de objetos educacionais, esse projeto provém do uso de Objetos de Aprendizagem Digital como ferramentas acessíveis e criadores de ambientes de aprendizagem via Web. Possibilitando assim, ser um recurso de ensino reutilizável.

Um corpo multidisciplinar trabalha na confecção do material contribuindo para fatores técnicos, gráficos e pedagógicos. Entretanto, mesmo salientando os profissionais que integram a produção, o principal objetivo aqui é determinar o quanto a condução projectual interfere no equilíbrio de um trabalho adequado e quão importante o planejamento técnico-gráfico-pedagógico se apresenta no decorrer do processo de produção de um OAD.

\section{DESENVOLVIMENTO DO OAD}

\section{Designer Pedagógico}

A principal intenção de um material instrucional digital, é instigar e criar um ambiente motivador onde o aluno construa o próprio conhecimento atuando com uma postura crítica, criativa, investigativa e autônoma. Características essas que devem ser contempladas no momento em que a metodologia estiver centrada no aluno e na sua ação sobre o ODA [2].

O Designer Pedagógico deve contribuir com o conhecimento para realização de um projeto que apresente um ambiente interativo, propiciando a motivação onde o aluno possa realizar relações de informações de forma multidisciplinar e colaborativa, onde é justificada pela teoria construtivista de Piaget, a qual fixa a interação interpessoal com colegas e professores, interatividade com conteúdo e diferentes fontes de informação.

Para isso, é necessário o planejamento onde a ferramenta funcione de maneira não linear, ou seja, ofereça a oportunidade de percorrer livremente o ODA. Além disso, o contato com situações aleatórias que causem instigações e necessita de usuais investigações e pesquisas vão permitir interação interpessoais - colegas e professores- e materiais didáticos (uso de hipermídia e hipertextos). Isso permitirá uma trajetória de busca criada pelo aluno, por isso uma navegação não-linear e que não conduza a uma lógica especifica [2].

\section{Designer Instrucional}

Enquanto o Designer Pedagógico se preocupa na elaboração de uma maneira eficaz de atingir o aluno de forma teórica e projectual no âmbito pedagógico, o Designer Instrucional elabora os métodos de instrução mais adequados a diferentes tipos de aprendizagem [5].

A habilidade de permear entre diferentes campos de conhecimento, permite ao profissional idealizar a forma e contextualizar a informação desejada à ferramenta que está sendo direcionada, conhecendo previamente o público ao qual será determinado o produto a ser desenvolvido.

Segundo [5] o Designer Instrucional é o responsável por projetar soluções para problemas educacionais específicos identificando as necessidades de aprendizagem e desenhar, desenvolver, implementar e avaliar uma solução para esse caso. Portanto, no ODA, o Designer Instrucional adéqua as qualidades pedagógicas as necessidades e define de maneira eficaz a utilização da tecnologia.

Apesar das características de aplicação de interface estarem a cargo do design gráfico, o designer instrucional deve pensar na utilização da tecnologia vigente no decorrer do projeto e apresentar maneiras praticas para a aprendizagem do usuário dentro do contexto e padrão de utilização da tecnologia.

"O designer instrucional também é responsável por apresentar e validar com o cliente e demais interessados os produtos resultantes de cada fase, apresentando relatórios de análise e acompanhamento, documentos de especificação, pilotos e avaliações" [5]

\section{CONFECÇÃO VISUAL DO ODA}

\section{Desenvolvimento visual do projeto}

A aplicação das diretrizes visuais no desenvolvimento de uma interface fica a cargo do designer gráfico responsável na elaboração e na adequação de princípios comuns à uma boa interface aplicando elementos como usabilidade e funcionalidade.

Segundo [3], uma interface pode inspirar sentimentos de afeição ou rejeição o que determina a maneira como o usuário se comportará frente a um sistema operacional. Diante disso, o mesmo autor cita que $86 \%$ das pessoas que abandonam o uso do programa, o fazem por causa da interface.

Então pode-se entender que o primeiro contato com a interface é um cuidado a se tomar, já que a primeiro experiência do usuário apresenta um passo importante na sua continuidade de uso.

\section{Interfaces Gráficas}

Segundo [21], as interfaces gráficas surgiram nos anos 80, substituindo as interfaces de texto, transformando a tarefa antes só destinada a especialistas em uma atividade mais simples, atendendo as necessidades dos leigos e usuários mais comuns.

Com o advento das interfaces gráficas, a imagem, elemento há muito exaltado na comunicação humana passa a integrar a comunicação homem máquina, assumindo o papel de signo icônico, representando ações dentro de uma atividade computacional.

Nessa conjuntura segue a necessidade de um estudo apurado na importância do signo e como ele atua na percepção humana para um desenvolvimento apropriado e de uma interface que atinja seu objetivo educacional.

Entretanto só não basta ter um visual agradável, segundo [17], o papel principal da interface é criar a interação do usuário e o computador transitando entre o real e o digital, apresentando aspectos como transparência, naturalidade, intuitividade e praticidade atendendo assim, os requisitos da ergonomia visual.

A Figura 1 ilustra um exemplo de uso dos ícones que exercem uma naturalidade na interação com o usuário eles são familiares ao público no qual se dirige a atividade e o layout exerce um ambiente de fácil navegabilidade.

\section{(C) 2014INTERTECH}

March16 - 19, 2014, Guimarães, PORTUGAL XIII International Conference on Engineering and Technology Education 


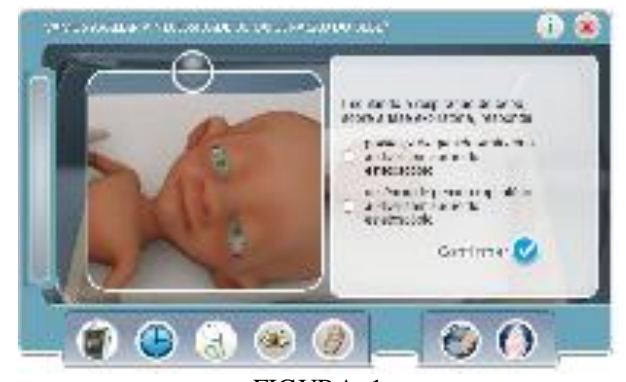

FIGURA. 1

JOGO "I BABY" PRODUZIDO PELA COMPANHIA ILUSTRADA

\section{Ergonomia visual}

A observação "homem $\mathrm{x}$ tarefa $\mathrm{x}$ maquina" foi um avanço obtido no decorrer da evolução tecnológica. Seguindo essa tendência evolutiva, surgiu a ergonomia, estudo que segundo [20] busca conhecer como os usuários percebem a tarefa a ser executada, como ocorre a interação homem máquina e o processo de conhecimento, fazendo assim, uma transposição do modelo mental para o sistema computacional. Assim, a Ergonomia de Software vem observar características como utilidade e usabilidade de interfaces digitais favorecendo a adequação das ferramentas visuais as tarefas e objetivos na interação do usuário [12].

\section{Interação e Semiótica: $O$ ícone e sua identificação na interface}

Um dos recursos que apoiam uma interface é a Semiótica. No intuito de valorizar espaços e o segmento da ordem visual, pode ser utilizada de forma que sacie a necessidade de uso de textos longos, substituindo por ícones ou imagens que simbolizem a ideia e reduza os textos escritos criando um ar de beleza e valorizando a interface.

O princípio do ícone, ilustrado na Figura 2, é a representação do objeto ou da ação a ser feita, por isso a valorização da simplicidade na fabricação de um ícone como [20] destaca, referindo-se também à preocupação com a representação em fator do tamanho no qual o signo é inferido e a necessidade de simplificar ao máximo fazendo com que a estética visual não subjugue ou prejudique a visualização do ícone.

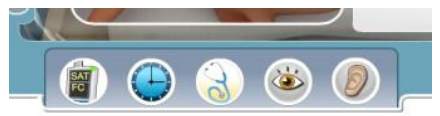

FIGURA. 2

JOGO "I BABY" PRODUZIDO PELA COMPANHIA ILUSTRADA

"Na teoria dos signos de C. S. Peirce, responsável por ter notabilizado o conceito de iconicidade, o ícone é um signo que significa seu objeto porque apresenta semelhanças qualitativas com ele. Ora, um texto é tanto mais literário quanto mais a linguagem, ao manipular o potencial icônico da língua, é capaz de materializar nas próprias palavras aquilo sobre o que fala, transfigurando a convencional idade em sentidos motivados que saltam à flor da pele das palavras. " [18]

\section{Carga cognitiva Visual na interface}

A memória humana tem a capacidade limitada de processamento. Nesse aspecto a interface não deve de forma alguma atrapalhar a informação passada no OAD, tentando sempre contribuir para a aprendizagem do usuário, inferindo de forma agradável na carga cognitiva e aprendizagem do indivíduo que utiliza o material.

Dado que há várias fontes de informação competindo por essa limitada capacidade, no aprendizado eletrônico precisamos apoiar o aluno nos processos de seleção, integração, armazenamento e recuperação da informação." [5]

O conceito central é minimizar a carga de trabalho cognitivo, incluir ideias claras e concisas de forma que, o tempo de leitura e a ocorrência de erros seja mínima. Dessa forma, o número de passos para realização de uma atividade ou ação devem ser reduzidos ao máximo, evitando assim numerosas e complexas ações para atingir um objetivo.

A densidade informacional deve ser equilibrada de modo a aumentar a performance do usuário no uso do OAD [12].

\section{Objeto de exemplo}

Aqui será abordado o conceito de navegação utilizando dois OADs de referência: "A viagem de Kemi", desenvolvido por um grupo multidisciplinar de estudos da Universidade Estadual Paulista Júlio de Mesquita Filho - UNESP, campus de Bauru e o "Mundo das letras", desenvolvido pela Companhia Ilustrada.

A Figura 3 ilustra a tela inicial deste OAD cujo objetivo é ensinar ao usuário conceitos químicos, utilizando diversos tipos ilustrados para estimular o aluno a conhecer mais sobre a presença dos elementos químicos no desenvolvimento da sociedade moderna.

Os elementos gráficos utilizados no início pois são bem organizados e distribuídos. A centralização dos objetos e cores não prejudicam a boa visualização do objeto.

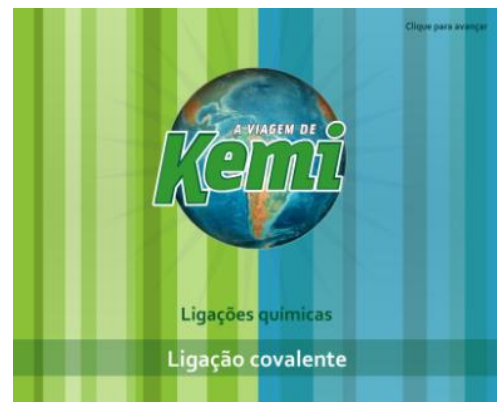

FIGURA. 3

A VIAGEM DE KEMI

Entretanto, em uma avaliação mais criteriosa percebe-se um problema para o avanço da atividade. A mensagem "Clique para avançar" no topo a direita não permite uma boa navegação e interação, exigindo do usuário uma atenção maior para identificar a atividade a ser exercida para o avanço da atividade.

Um meio de auxílio à navegação seria o uso de um botão iniciar ou aumentar a fonte da mensagem, diminuindo assim o esforço do usuário.

Atendendo alguns requisitos baseados nos termos da usabilidade como intuitividade e eficiência permitindo uma rápida e 
fácil memorização, o ideal seria o uso de um botão maior e símbolos mas generalizados, evitando assim, cansaço intelectual do usuário.

$\mathrm{O}$ uso de uma fonte de corpo pequeno e uma cor que não oferece destaque, não auxilia a navegação, prejudicando assim, o seguimento da atividade.

Adiante é feita a distinção das alterações a serem seguidas para auxiliar a navegação do OAD.

- Alteração de cor;

- Aumento da fonte;

- Alteração da diagramação de modo que o layout auxilie na navegação.

- Uso de símbolos comuns e confecção de um botão diferenciado do cenário que permita a ação de iniciar ou avançar a atividade.

A Figura 4 representa uma boa utilização da navegação. Embora seja voltado a outro público e com faixa etária diferente, o cuidado com o layout deve ser feito da mesma forma.

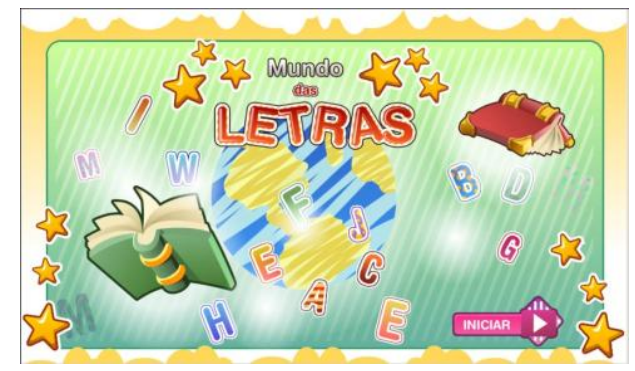

FIGURA. 4

MUNDO DAS LETRAS, PRODUZIDO PELA COMPANHIA ILUSTRADA

Estendendo para o restante do objeto, conseguimos averiguar a navegabilidade facilitada pelo contínuo uso de cores e formas comuns nos botões de navegação do objeto. Abaixo a exemplificação em várias fases do ODA Mundo das letras, Figura 5.

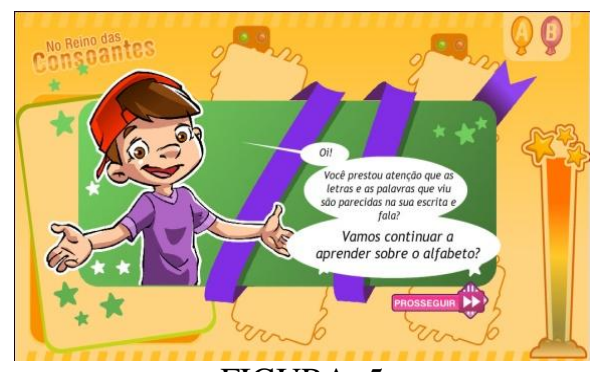

FIGURA. 5

MUNDO DAS LETRAS, PRODUZIDO PELA COMPANHIA ILUSTRADA

Baseando-se nas heurísticas de [13], onde são descritos os critérios básicos para a usabilidade, os OAD Mundo das letras e Viagem de Kemi apresentam algumas disparidades funcionais no quesito intuitividade tais como a não diferenciação dos objetos na tela de introdução, já descrito acima e que prossegue no restante do objeto.

Na segunda tela da Viagem de Kemi, Figura 6, observa-se a mesma falha de navegação onde não há destaque no botão de avanço da tela, prejudicando o que [13] destaca como qualidade extra chamada de facilidade de entendimento.

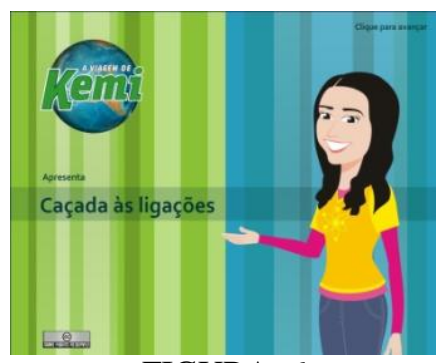

FIGURA. 6

A VIAGEM DE KEMI,

A ausência de destaque prejudica a ação do usuário e interfere em sua atividade cognitiva forçando o usuário a direcionar uma atenção a uma ação que deveria ser intuitiva e assim, prejudicando sua eficiência na atividade.

\footnotetext{
" O sistema deve apresentar facilidade de uso permitindo que, mesmo um usuário sem experiência, seja capaz de produzir algum trabalho satisfatoriamente". [13]
}

\section{DIRETRIZES PARA A ELABORAÇÃO VISUAL DE UM OBJETO DE APRENDIZAGEM DIGITAL}

\section{Cores}

O aspecto visual deve ser firmado sobre cores que não prejudiquem o intuito pedagógico e não atuem de forma negativa no processo cognitivo da aprendizagem.

$\mathrm{O}$ uso de cores no aspecto geral devem se apresentar como um suporte ao material não devendo assim, comprometer o conteúdo pela forma visual adotada.

Segundo [15], não existe uma definição específica para o uso de cores em interfaces homem x computador, o que tem sido feito é a adaptação dos conceitos e princípios aplicados para o design gráfico de revistas, livros, televisão, dentre outros.

A cor apresenta-se como uma importante propriedade estética na interface, pois pode ser utilizada para identificar elementos que devem atrair a atenção do usuário.

$\mathrm{O}$ uso indevido das cores pode trazer consequências negativas prejudicando a produtividade e a motivação do usuário na atividade exercida.

Cores brilhantes, vívidas e fortes contrastes atraem a atenção do observador, mas podem confundir ou dispersar o usuário dificultando a legibilidade da mensagem visual.

A cor pode auxiliar o processo visual e cognitivo da informação, associando-a a significados que representam

[15] apresenta os seguintes estereótipos naturais da cultura ocidental para o significado das cores:

- vermelho: perigo, atenção, calor e comandos de interrupção;

- $\quad$ amarelo: cuidado, teste e lentidão;

- $\quad$ verde: passagem livre, normalidade e segurança;

- laranja: valor limite e radiação;

- $\quad$ azul: frio, água, céu e calma;

- cinza, branco e azul: inatividade, neutralidade.

Cores quentes: ação, resposta requerida e proximidade (cores quentes são aquelas associadas a ideia de sol, fogo, etc., como: o amarelo alaranjado, o vermelho, dentre outras). 
Cores frias: distância e informação de fundo (cores frias são aquelas associadas ao verde-azul da água, que dá a sensação de frio, como: o amarelo-verde, o verde, o azul, o violeta, dentre outras).

Considera-se então, o uso de cores frias para fundos e objetos que tenham muito texto afim de não prejudicar a leitura e atenuar o cansaço do usuário.

Para botões ou mensagens rápidas o uso de cores ditas "quentes" devem ser utilizadas para ambientar a importância da mensagem ou diferenciar-se dos objetos da interface, tal como objetos de clique e mensagens de ajuda ou informações sobressalentes do ODA.

Entretanto [15] faz recomendações sobre o uso de cores em interfaces:

- O número de cores em uma interface deve ser limitado, formando um conjunto de cores padrão que poderá ser modificado ou recuperado pelo usuário de acordo com suas necessidades;

- As cores quentes devem ser usadas com moderação para melhorar a focalização dos elementos;

- o Devem ser utilizadas no mínimo duas e no máximo cinco cores diferentes em uma interface.

- A cor não deve ser o único elemento usado para distinguir informações, formas, rótulos e agrupamentos devem exercer um papel complementar;

- As cores neutras para o fundo realçam a visibilidade dos elementos em primeiro plano. $\mathrm{O}$ agrupamento de elementos, quando determinado pelo uso de cores, deve manter a consistência, não utilizando a referida cor para elementos que não estão relacionadas àquele agrupamento;

- A relação e associação de cores a significados, pode variar conforme a cultura ou indivíduo;

\section{Fundos}

Segundo [12], na comunicação visual as relações entre figura e o fundo são de extrema importância para o sucesso da informação, pois de sua distinção depende da clareza da mensagem.

No ODA o plano de fundo devem apresentar cores que diferenciam-se dos elementos dispostos na interface, para que não haja ruído e não prejudique a simplicidade e a clareza da comunicação.

Segundo [15] a composição de fundo para uma Interface é composta por padrões de texturas e/ou cores, sua escolha está totalmente ligada ao aumento do interesse do projeto visual e para que este fundo esteja em sintonia com o restante do elementos, o mesmo deve seguir as seguintes recomendações e observações sobre cores:

- Utilizar cores neutras para o fundo onde possui textos;

- Não utilizar cores muito escuras para o fundo de toda a interface, pois isso aumenta o cansaço visual do usuário. Em pequenas áreas, estas cores podem ajudar a direcionar a atenção do usuário;

- Não dispor de elementos como fundo sem planejamento, os elementos devem estar em harmonia com todo o conteúdo.

- Para fundos coloridos, os textos devem estar em perfeito contraste com o fundo, de modo a obter a visibilidade elegibilidade do texto.
- Numa interface, o fundo não deve se destacar mais que a informação, deve-se fazer uso de elemento cromático (texto, ícones, figuras, etc.) sobre fundo acromático (branco, preto e cinza) e vice versa.

\section{CONSIDERAÇÕES FINAIS}

A análise dos parâmetros de design para construção de um ODA permitem um avanço na possibilidade de entender a construção pedagógica e a metodologia a serem tomadas.

Todos os processos aqui abordados trouxeram à tona a dificuldade da elaboração de um ODA desde a sua fase embrionária até a fase final onde se encontra a abordagem sistemática do objeto de forma visual.

É de extrema importância o estudo e o conhecimento dos termos da usabilidade, entretanto, as infinitas possibilidades tornam necessária a análise individual de cada objeto e exige esforços para que cada OAD desenvolvido seja realizado de forma efetiva e eficaz.

\section{REFERÊNCIAS}

[1] BARBOSA FILHO, André. CASTRO, Cosette. Comunicação digital: educação, tecnologia e os novos comportamentos. São Paulo: Paulinas, 2008.

[2] BEHAR, P. A, TORREZZAN, C.A.W, Metas do design pedagógico: um olhar na construção de materiais educacio-nais digitais. 2009.

[3] BELTRÁN, B. An EPSS. Interface that people can use. Disponível em http://ourworld. compuserve.com/homepages/bea beltran/

[4] Cybis, W.A., Betiol, A., Faust, R., Ergonomia e Usabilidade: Conhecimentos, Métodos e Aplicações, Jump Editora, São Paulo, 2007, p350.

[5] FILATRO, A., Design Instrucional na prática. Pearson / Prentice-Hall, 2008.

[6] Gangwer, Timothy Patrick, Visual Impact, Visual Teaching: Using Images to Strengthen Learning, Publisher Corwin Press, 2005. Editora SENAC.

[7] HIRATSUKA,T. P. Contribuições da Ergonomia e do Design na Concepção de Interfaces Multimídia.1996, UFSC - Universidade Federal de Santa Catarina, Florianópolis Disponível em: http://www.eps.ufsc.br/disserta96/tei/index/index.htm

[8] KENSKI, Vani M. Educação e Tecnologias o novo ritmo da informação. Campinas, Papirus, 2007.

[9] KRUG, S. Não me faça Pensar. Alta Books. Rio de Janeiro, 2011.

[10] LEMOS, J. G; WINCK, J. B. Design de Relações entre tecnologias amigáveis e sociabilidades virtuais: um novo campo de investigação para o Design. XXXIII Congresso Brasileiro de Ciências da Comunicação - Caxias do Sul, RS INTERCOM, 2010

[11] LITTO, F.; FORMIGA, M. Educação a distância: o estado da arte. ABED, Prentice Hall: São Paulo, 2008.

[12] LORDELO, Antônio Luis, Interface Gráfica e educação a distância, 2004

[13] Nielsen, Jakob, Usabilty Engineering, Morgan Kaufmann, Inc. San Francisco, 1993.

[14] Nielsen, Jakob. TV Meets the Web. Useit. Disponível em: <http://www.useit.com/alertbox/9702b.html>. Acesso em: 10/11/2011. 2009.

[15] PARIZOTTO, R. Elaboração de um guia de estilos para serviços de informação em ciência e tecnologia via web. 1997. Dissertação (Mestrado em Engenharia da Produção) - Universidade Federal de Santa Catarina, Florianópolis. Disponível em <http://www.eps.ufsc. br/disserta98/rosam>. Acesso em: 21 set. 2002.

[16] Pazos-Arias, J. J. et al. ATLAS: a framework to provide multiuser and distributed tlearning services over MHP. Software: Practice and Experience. 2006.

[17] RADFAHRER, L. Design/web/design. São Bernardo do Campo: Market Press, 1999

[18] SANT AELLA L. - O que é semiótica. São Paulo: Brasiliense, 1983

[19] SERVIÇO NACIONAL DE APRENDIZAGEM INDUSTRIAL. Departamento Nacional. Impactos ocupacionais e educacionais da TV digital no Brasil. Brasília, SENAI/DN, 2008

[20] SILVA, C.A.P. Qualidade da comunicação iconográfica no ambiente informatizado de trabalho. 1996. Tese (Doutorado em Engenharia da Produção) - Universidade Federal de Santa Catarina, Florianópolis. Disponível em: http://www.eps.ufsc.br/teses96/pereira/cap3/capitulo3.htm

[21] STEFANELLI. Eduardo José. A importância do profissional de comunicação gráfica na produção de material para EAD. Disponível em http://www.abed.org.br/texto04.doc

[22] TUFTE, E. The Visual display of quantitative Information. Second Edition. Graphicas Press LLC. Cheshire, Connecticut, USA. 2007

[23] VALENTE, Vânia C. P. N. Representação Gráfica na produção de material instrucional para TV Digital - GRAPHICA'2009 - XIX Simpósio Nacional de Geometria Descritiva e Desenho Técnico e VIII International Conference on Graphics Engineering of Arts and Design. 2009 